Revue d'histoire de l'enfance « irrégulière »

Le Temps de l'histoire

$8 \mid 2006$

Le corps du délinquant

\title{
Heather Shore, Artful Dodgers : Youth and Crime in early 19th century London
}

John Ward

\section{OpenEdition}

Édition électronique

URL : http://journals.openedition.org/rhei/388

DOI : 10.4000/rhei.388

ISBN : 978-2-7535-1646-5

ISSN : $1777-540 \mathrm{X}$

Éditeur

Presses universitaires de Rennes

Édition imprimée

Date de publication : 15 novembre 2006

Pagination : 188-191

ISSN : 1287-2431

\section{Référence électronique}

John Ward, " Heather Shore, Artful Dodgers : Youth and Crime in early 19th century London ", Revue d'histoire de l'enfance «irrégulière » [En ligne], 8 | 2006, mis en ligne le 06 février 2007, consulté le 04 décembre 2020. URL : http://journals.openedition.org/rhei/388; DOI : https://doi.org/10.4000/rhei 388

(C) PUR 
Heather Shore

Artful Dodgers : Youth and Crime in Early Nineteenth-Century London Woodridge, The Boydell Press, 2002, 193 p.

Cet ouvrage d'une historienne universitaire, dont la première édition date de 1999, se propose d'étudier l'émergence de la catégorie des " délinquants juvéniles " au cours de la première moitié du XIXème siècle. L'auteure compare les représentations de ces mineurs véhiculées par les autorités publiques, les réformateurs et dans l'opinion publique avec les réalités de leur vie dans la rue et de leur traitement par le système judiciaire et au sein des établissements pénitentiaires. Le discours de l'époque est analysé à partir des nombreux écrits polémiques, littéraires et politiques des philanthropes, parlementaires et observateurs, tels que Mary Carpenter (philanthrope militante de la cause des enfants) ou Henry Mayhew (auteur d'une des premières enquêtes sociales). Les sources primaires étudiées (registres publics, dossiers de police, carnets de notes des premiers observateurs sociaux) permettent d'établir un inventaire très complet des conditions qui prévalent à Londres. Par ailleurs, l'auteure met en évidence les conditions d'extrême rudesse qui règnent à la colonie pénitentiaire de Tasmanie et dans la prison bateau l'Euraylus, ces conditions étant saisies à travers une source d'une valeur exceptionnelle : les retranscriptions d'entretiens menés sur des enfants détenus par un commissaire de police chargé à l'époque d'une investigation officielle, et les notes personnelles de celui-ci.

Le titre, Artful Dodgers, est tiré du célèbre roman de Charles Dickens, Oliver Twist. Il correspond à l'image d'Épinal du voleur à la tire capable de mener son " art " avec brio et de fuir (to dodge) le bras armé de la loi avec célérité. Nous pouvons découvrir une gravure de Georges Cruickshank (1792-1878), illustrateur de l'œuvre de Dickens de l'époque, en page de garde. Le dodger personnifie le stéréotype populaire du délinquant, vu dans l'imaginaire collectif comme un "professionnel ", appartenant à une " race " de jeunes ayant de fortes propensions au crime et à la récidive et appartenant à des bandes bien organisées. À noter que jusqu'en 1808, le vol à la tire est passible de la peine de mort. Si le voleur mineur est systématiquement gracié,

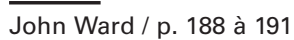


la symbolique de la peine et l'occasion qu'elle procure au jeune détenu d'entrer dans une " école du crime " contribuent à renforcer la représentation populaire d'un jeune criminel voué inévitablement à une carrière criminelle et appelant une peine d'une extrême sévérité.

Outre la figure du dodger, l'auteur propose une analyse détaillée des classifications des jeunes, fondées sur une opposition récurrente entre cette figure du « criminel d'habitude " et celle du criminel "victime " de son milieu domestique instable, mais néanmoins récupérable. Ainsi, la Société philanthropique différencie les " orphelins », la descendance «non délinquante » de parents incarcérés et les vrais « délinquants »; ceux appartenant aux deux premiers groupes sont placés dans une école industrielle, The Manufactory, les derniers dans un établissement au régime plus sévère, The Reform. L'on oppose aussi les "bons garçons » aux "garçons endurcis ", les " délinquants travailleurs volontaires ", ceux " qui refusent de travailler " à ceux " qui ne peuvent travailler ", etc. Cette rhétorique passe par bien des formes pour aboutir à une nouvelle terminologie, employée plus systématiquement vers la fin de la période, qui sépare les garçons " endurcis " (hardened) de ceux qui "périssent" (perishing).

Le concept de délinquance juvénile constitue ainsi, selon l'auteure, une véritable « invention » de l'ère victorienne, et les causes de cette délinquance font l'objet de nombreux débats. Les analystes de l'époque, sans cesser de faire appel aux facteurs moraux si familiers - non observance de la religion, conduite immorale des parents, vie dissipée favorisée par l'urbanisation... -, adoptent désormais une perspective plus sociologique, évoquant le déclin de l'apprentissage et ses effets sur la composition de la famille, l'impact du système policier et pénal lui-même, ou encore la sociabilité en bande des délinquants qui s'agrègent dans les pubs et leur dérivés, les flash houses, bars semi clandestins servant de lieu d'initiation aux activités criminelles dans les bas quartiers.

Laction des philanthropes a également contribué à la hausse des chiffres de cette délinquance en encourageant les victimes à déposer plainte, sachant que les peines seraient moins sévères qu'auparavant. Face à la vague de délinquance juvénile, l'introduction de formes de comparution immédiate (summary jurisdiction) durant la décennie 1820 a sans doute contribué à plonger de nouvelles franges de la population des enfants errants dans un processus de criminalisation. 
Contrairement à l'opinion largement répandue, la majorité des mineurs concernés combinaient une activité légitime avec des vols occasionnels. La complexité de leurs motivations apparaît à travers les récits de la vie dans la rue, les plaisirs du jeu, des théâtres à un sou et de la drague. Selon Heather Shore, les garçons ne voyaient pas nécessairement le vol comme une activité différente de celle de leurs autres comportements adolescents. Un détenu de l'Euraylus dit : «Il n'est pas surprenant que les garçons ne se réforment pas s'ils ont un peu de succès, le charme [ $d u$ vol] c'est que ça vient si facilement et ça s'en va aussi vite, c'est beaucoup mieux que de travailler dur » (p. 56). Il n’y avait pas de frontière nette entre l'adolescence des classes défavorisées et celle des délinquants.

L'évolution du système judiciaire est marquée aussi par la reconnaissance progressive d'une responsabilité pénale atténuée des mineurs et par l'émergence, à la fin de la période, de peines supposées contribuer à leur réforme et non seulement à leur punition. L'incapacité juridique, dite "Doli Incapax " (principe, proche du concept français de discernement, selon lequel il faut apporter la preuve de la capacité du mineur à se rendre compte de sa culpabilité), est appliquée aux très jeunes enfants depuis le XVIème siècle. Il faudra attendre la période qui succède à celle étudiée par l'auteure pour que cette conception soit consolidée et intégrée dans la législation britannique, qui s'avère particulièrement complexe.

Outre sa contribution à l'étude des débats de fond, l'ouvrage fournit de nombreux éléments descriptifs relatifs aux conditions de vie des enfants, aux subtilités des distinctions juridiques et jargonnesques entre les différents actes délinquants (une tentative d'effraction par la fenêtre d'un magasin se dit « faire une étoile sur la glace» (starring the glaze) et la punition est plus ou moins sévère selon que le jeune a passé son bras à travers la fenêtre ou non...), à la politique d'application des peines (d'une effrayante sévérité) et aux conditions de détention. Le lecteur pourra se rendre compte de l'état d'esprit de certains détenus juvéniles, de leur insouciance apparente face aux perspectives de punition (notamment par la déportation) et de leur souffrance au sein des établissements pénitentiaires. Des citations de lettres de parents implorant la clémence permettent de démentir l'opinion répandue selon laquelle l'environnement familial fut systématiquement négligeant ou néfaste.

Malgré la minutie de présentation des sources et la complexité des thèmes 
abordés, cet ouvrage reste d'une lecture agréable et instructive. Pour le lecteur peu familiarisé avec la civilisation anglaise, il sera parfois difficile de se repérer géographiquement et institutionnellement parmi les nombreuses instances citées. L'on peut regretter aussi l'absence d'explications et d'analyse des principes juridiques alors en cours, ce qui rend difficile de saisir la portée des lois importantes votées au cours des années 1820 , relatives au vagabondage notamment. En dépit de ces inconvénients mineurs, l'ouvrage constitue sans aucun doute une contribution majeure à la recherche historique et offre, en outre, des perspectives de comparaison avec le temps présent à travers des thèmes tels que la "comparution immédiate ", les attitudes face aux enfants meurtriers et à leur traitement, ou encore l'analyse de la frontière si difficile à tracer entre le comportement "antisocial » et la « délinquance avérée ». Il s'agit d'un ouvrage à recommander fortement à tout lecteur, spécialiste ou non, intéressé par ce champ d'étude.

John Ward 